\title{
Evaluación Institucional
}

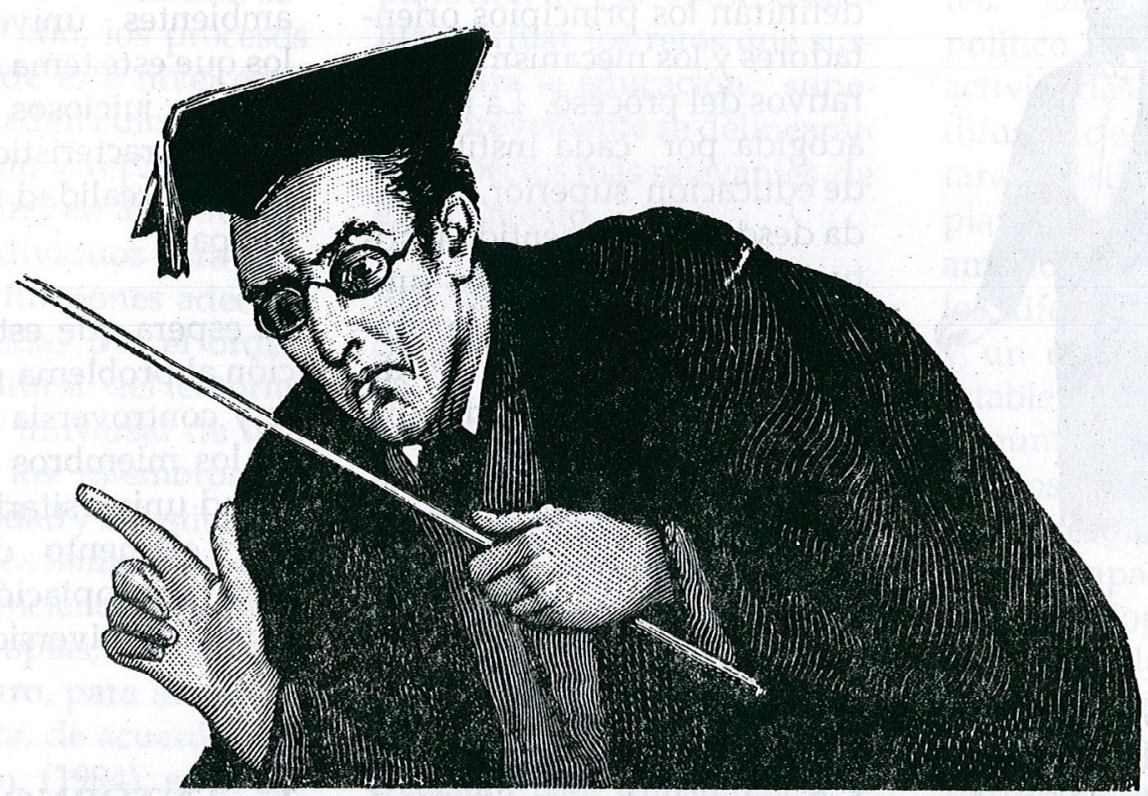

\section{AUTOEVALUACION INSTITUCIONAL EN LA PERSPECTIVA DE LA AUTORREGULACION: FUNDAMENTO PARA LA AUTONOMIA UNIVERSITARIA*}

Nohra Pabón Fernández

Profesora Facultad de Educación

Universidad Pedagógica Nacional.
$\mathbf{L}$ as instituciones de educación superior del país serán ahora protagonistas de la creación y aplicación de teorías, metodologías y procedimien- tos de evaluación y desarrollo institucional, concebidas para ellas y desde ellas mismas.

A raíz de la expedición de la Constitución de 1991, y habien-
* Documento presentado en el Seminario-Taller "La acreditación universitaria en el contexto internacional: tendencias, problemas y alternativas de solución". Universidad de Bogotá Jorge Tadeo Lozano. Santafé de Bogotá, mayo de 1995. 


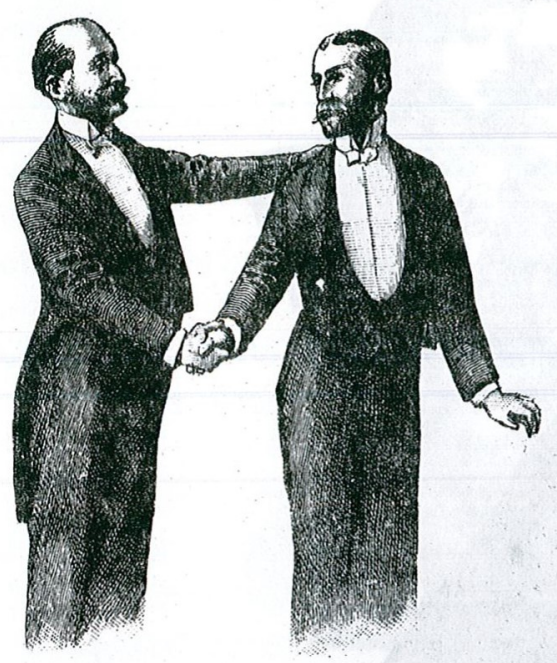

do la ley 30 de 1992 creado al Sistema Nacional de Acreditación con el objeto de "garantizar a la sociedad que las instituciones de educación superior que hacen parte del sistema, cumplan los más altos requisitos de calidad y que realizan sus propósitos y objetivos", estas instituciones se ven abocadas a la necesidad de desarrollar estrategias y mecanismos que les permitan mostrar al Estado y a la sociedad la calidad con que asumen los procesos educativos.

La autoevaluación es la base fundamental del proceso de acreditación. Con ésta se inician los diferentes procesos evaluativos que finalmente permiten, al Consejo Nacional de Acreditación, recomendar o no la acreditación de una universidad. La autoevaluación es el elemento nuclear de todo el proceso de acreditación; para su realización, las instituciones definirán los principios orientadores y los mecanismos operativos del proceso. La misión acogida por cada institución de educación superior, definida desde su ser y sentido institucional, y el logro de esas funciones con excelencia, posibilita la concreción particular de parámetros de calidad para cada institución.

Las universidades tendrán que construír un modelo de autoevaluación que, además de permitirles establecer el grado de coherencia entre lo que han definido que son o quieren ser, y lo que efectivamente logran, deberá constituírse en el elemento dinamizador de un proceso permanente de cambio o de ajuste, orientado a la búsqueda de la calidad, en el marco de las intenciones explicitadas en su proyecto educativo institucional y en su misión.

El problema de la autoevaluación y acreditación requiere un proceso de asimilación de teorías, procedimientos y técnicas y de construcción de estrategias para su implementación, entre otras cosas. Para esta tarea, nadie más llamado a participar que las mismas comunidades académicas de las universidades.

La creación de una cultura de la autoevaluación y de la acreditación es un primer paso en la búsqueda y construcción de ambientes universitarios, en los que este tema sea objeto de aportes juiciosos y asentados en las características y dinámica de la realidad universitaria del país.

Se espera que esta aproximación al problema genere debate y controversia constructiva en los miembros de la comunidad universitaria, como primer elemento dinamizador para la apropiación de este tema en la universidad.

\section{ENTORNO YRETOS DE LA EDUCACION SUPERIOR PARA EL SIGLO XXI}

La educación superior de finales del siglo $X X$, transcurre en un entorno que rápidamente acumula demandas educativas de diverso orden. La universidad, se encuentra inmersa en las condiciones dinámicas y cambiantes de la sociedad que le da origen; la cultura propia del entorno, tomada ésta en su más amplia acepción, y la desarrollada por la humanidad, configuran el universo objeto del conocimiento de la educación superior.

Trascender el conocimiento acumulado y generar una posición y una acción crítica y 
constructiva frente a la realidad, son tareas propias de las instituciones de educación superior. Por ello, los procesos educativos de este nivel educativo no pueden culminarcon la asimilación y reproducción de esa cultura, en aras de formar a los individuos para asumir roles y funciones adecuadas y definidas por el orden social y cultural del entorno particular o universal de una institución; los miembros de una universidad y la institución misma son los llamados a ejercer esa conciencia crítica, a construir utopías, a caminar hacia el futuro, para lo cual la vía más corta, de acuerdo con Tünnermann, (1994), es el estudio del pasado.

La cita que hace el mismo autor de Alfred Whitehead, la "universidad es también imaginación o no es nada, y su tarea es la creación del futuro", es otra forma de expresar esta expectativa acerca de la Universidad.

En este final de milenio, varias revoluciones confluyen en la configuración de una dinámica cultural que, en la mayoría de los casos, ha superado la capacidad de asimilación y transformación de las universidades. Existe un desequilibrio entre el quehacer de las instituciones de educación superior y lo que demandan las dinámicas de la sociedad, de la cultura, del mundo producti- vo $\mathrm{y}$ las mismas necesidades del desarrollo humano. La exploración de este entorno facilita perfilar los retos que surgen para la educación superior; brevemente se delinearán los aspectos más relevantes de este contexto.

El conocimiento, en las últimas décadas del siglo $X X$, ha logrado un papel estratégico en el concierto de las políticas para el desarrollo. Se ha convertido en factor de poder: más que la posesión de recursos naturales, que una ubicación ventajosa en el contexto geográfico, que la venta de mano de obra barata, una serie de factores como la creación, uso, transferencia y comunicación del conocimiento ha cambiado la forma y los medios de generación de la riqueza, y en consecuencia, las condiciones de vida y la calidad de la misma de los pueblos (Toffler, 1992). El conocimiento se cotiza con el valor de una mercancía. Entre los bienes económicos más preciados, se encuentra el carácter endógeno de las fuentes de conocimiento, dentro de las cuales es de destacar la producción y acumulación de conocimiento ( $\mathrm{Te}$ desco, 1993).

La orientación de la estrategia central definida en el documento de la CEPAL-UNESCO, (1992). Educación y conocimiento, eje de la transformación productiva con equidad, recoge desde esa perspectiva, propuestas para los países de la región en diferentes ámbitos. "Desde el punto de vista político, se trata de asumir las actividades de producción y difusión de conocimiento como tareas estratégicas de largo plazo que requieren el más amplio consenso posible entre los diferentes actores sociales, $y$ un compromiso financiero establecon su desarrollo; desde el punto de vista de los contenidos, de focalizar la acción en los resultados de la educación, la capacitación y la ciencia y tecnología, y en su articulación con las exigencias del desempeño de las personas, las empresas y las instituciones en los diferentes ámbitos de la sociedad; desde el punto de vista institucional, de romper el aislamiento de los establecimientos educativos y de generación y transmisión de conocimientos, e introducir modalidades

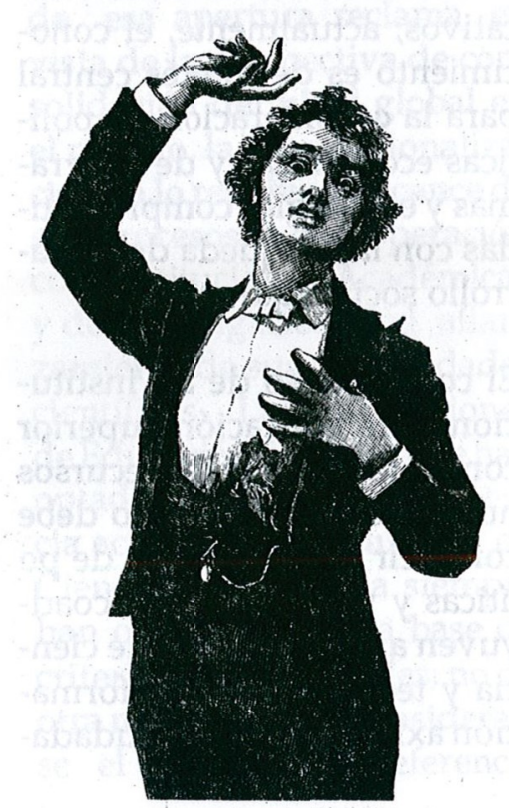




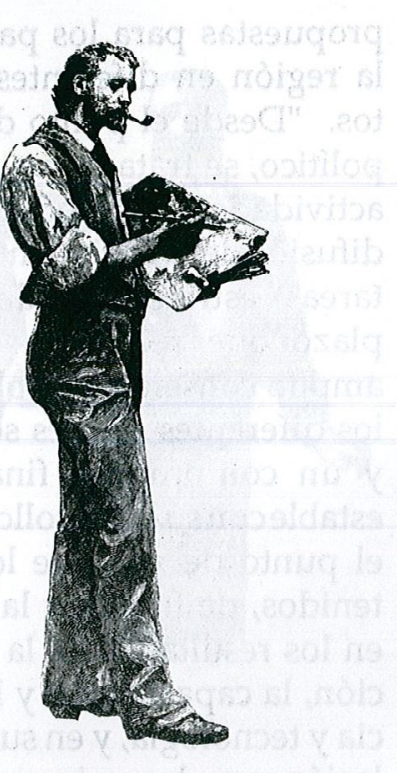

de acción en que los actores tengan mayores márgenes de autonomía en las decisiones, así como mayor responsabilidad por los resultados".

Las consideraciones sobre el conocimiento ya no son objeto exclusivo de los sistemas educativos; actualmente, el conocimiento es de interés central para la consideración de políticas económicas y de programas y estrategias comprometidas con la búsqueda del desarrollo social equitativo.

El compromiso de las instituciones de educación superior con la formación de recursos humanos y el desarrollo debe conducir a la definición de po líticas y programas que coadyuven a la generación de ciencia y tecnología y a la formación axiológica de los ciudada- nos que la modernidad reclama. La universidad tiene el reto de aportar a la formación de una sociedad con capacidades endógenas para el aprovechamiento científico-tecnológico, requisito para una presencia competitiva en el ámbito internacional (Brunner, 1993) y para aportar decididamente a la construcción de una sociedad justa y productiva.

La calidad de la educación, ahora más que nunca, deja de estar asociada a la acumulación de información; la formación integral del ser humano, y en lo relativo al conocimiento, su formación como individuo capaz deasimilar, transformar, crear y asumir una postura crítica y autónoma frente al mismo, son objetivos prioritarios a lograr. Un individuo capaz de enfrentar autónomamente los conocimiento, la ciencia, los saberes, la técnica y las responsabilidades éticas consigo mismo y con la sociedad puede integrarse como elemento dinamizador en la consolidación de una sociedad equitativa e independiente.

Las políticas de ofrecer cubrimiento, sin consideraciones mayores acerca de la calidad de la educación que se imparte, no tienen ninguna aceptación. La educación superior debe ser decalidad, debe cumplir con requirimientos universales si se quiere que sus egresados aporten decidida- mente a los procesos de desarrollo social y económico de sus naciones.

Los "trabajadores simbólicos", en términos de $W$. Reich, son los llamados a conservar un papel activo en los procesos productivos. La sistematización y robotización invaden espacios de trabajo y desempeño antes reservados para individuos que habían sido preparados en instituciones de educación superior para ello. En las instituciones universitarias, cada vez más, el paradigma de la educación debe afirmarse en el dominio de lo simbólico y en los potenciales de creación a partir de ello (Presidencia de la República, 1995); tal vez esto es lo que ha perdido la universidad, como nota esencial. La instrucción en el manejo de procesos y procedimientos, en la aplicación de fórmulas matemáticas y estadísiticas y otras actividades similares debe dejar paso a la formación del pensamiento, a la apropiación de los fundamentos de las ciencias y las disciplinas, a la comprensión de la problemática social y cultural del hombre y de la sociedad y al estímulo a la creatividad.

El crecimiento acelerado del bagage científico y tecnológico, también colocan en un plano secundario el propósito educativo de asimilar información. Aprender a aprender, a seleccionar información per- 
tinente, a utilizar los dispositivos tecnológicos de la informática y la comunicación y una actitud de renovación permanente frente al saber, constituyen los logros básicos con los que debe comprometerse la universidad en el propósito de lograr una formación integral. La educación permanente será una demanda de los egresados de la educación superior conscientes de la continua movilidad del conocimiento; cada vez más, los diplomas de terminación de estudios a nivel universitario, no serán más que un certificado de que un individuo ha sido preparado para que siga aprendiendo en el futuro. (Coombs, 1991).

El quehacer de las instituciones de Educación Superior, de alguna manera, se desarrollará en el Siglo XXI, a partir de la incorporación de las innova- ciones tecnológicas en el campo de la información y la comunicación. Por el tipo y dimensión de estas transformaciones, estas innovaciones constituyen una de las revoluciones que permiten hacer frente a los desafíos actuales de la Educación. En el año 2.000, cerca de 1.000 millones de habitantes de los 6.000 que tiene el planeta, estarán en capacidad de comunicarse entre sí de manera instantánea. (Tunnermann, 1994) La Universidad del Siglo XXI tendrá que incorporar estas tecnologías desde su perspectiva propia; concepción, procedimientos y metodologías para su utilización adecuada esperan la definción particular de las Instituciones de Educación Superior.

La flexibilización de sus estructuras y de sus métodos de trabajo permitirá a las univer-

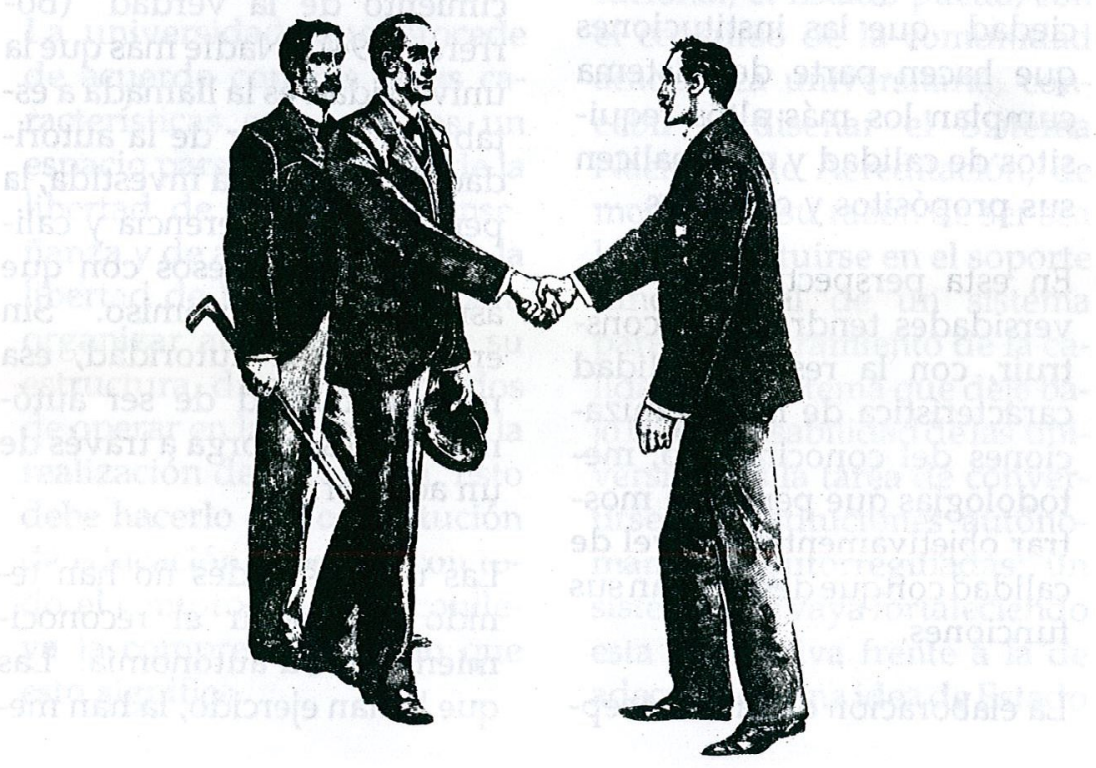

sidades adecuar permanentemente su quehacer a la definición utópica del futuro que ellas mismas se hayan planteado. Posiblemente también se constituirán en Centros de Formación y de actualización permanente del saber, en los que el desarrollo del ser humano, el servicio a la sociedad que les dio origen y la búsqueda permanente de conocimientos y técnicas sean el núcleo de sus preocupaciones y justificación de su quehacer. Las Universidades deberán proceder como organizaciones que aprenden y como entes en continua transformación, que no sólo responden sino que se adelantan a la dinámica de la realidad.

La universidad, más que cualquier otra institución, mantendrá su carácter como tal, si se configura como una organización abierta. Y la dimensión de esa apertura reclama, en vista de la perspectiva de consolidación del nivel global en el mundo, la internacionalización en lo relativo al alcance de sus procesos, a la cooperación con Instituciones Académicas y de Investigación y al afianzamiento de sus comunidades científicas. Las instituciones de Educación Superior que han optado por tener una presencia activa en la producción de Ciencia y Tecnología siempre han operado sobre la base de criterios internacionales; no de otra manera puede considerarse el entorno de referencia 


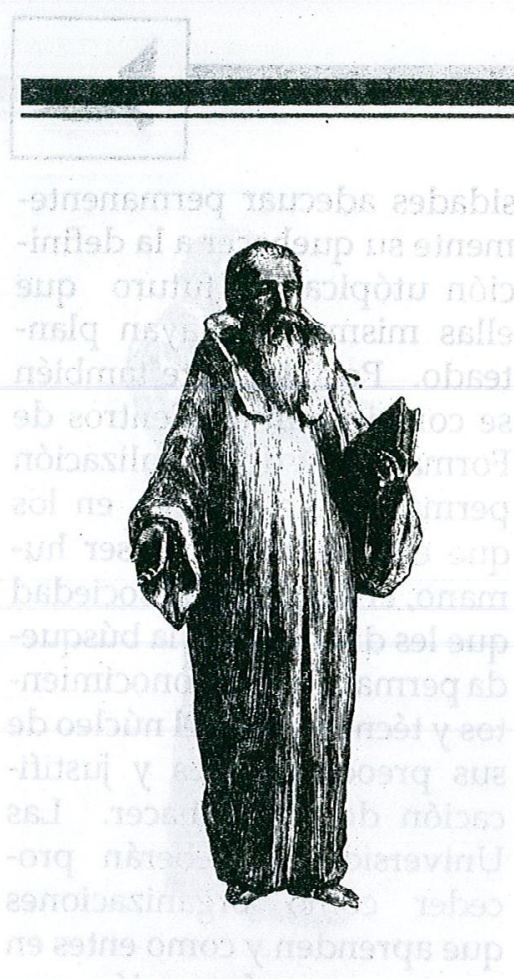

cuando se trata de producción de conocimientos.

\section{LAS REFORMAS DE LA EDUCACION SUPERIOR. EL CASQ DE LA ACREDITACION}

En el país, a raíz de la promulgación de la carta constitucional de 1991, y probablemente hubiese sucedido independientemente de ella, se ha venido revisando la legislación sobre la educación en general y, específicamente, sobre la educación superior. Punto nuclear de la Carta en este aspecto es la consagración, o mejor, el reconocimiento de la Autonomía Universitaria. Sus alcances y límites son definidos, en forma no muy clara, en la Ley 30 de 1992. A la vez, la Constitución asigna al Estado la función de inspección y vigilancia de la Educación; al ser ésta concebida como servicio público, le corresponde al Estado dar garantía de la calidad con que se imparte.

La misma Ley 30 delegó esta función del Presidente en el Ministro de Educación Nacional, para que fuese ejercida con la inmediata asesoría del Consejo Nacional de Educación Superior (CESU) y con la cooperación de las comunidades académicas, científicas y profesionales, de las entidades territoriales y de aquellas agencias del Estado para el desarrollo de la ciencia, de la tecnología, del arte y de la cultura.

Además, la Ley crea el Sistema Nacional de Acreditación (SNA), cuyo objetivo fundamental es garantizar a la Sociedad que las instituciones que hacen parte del sistema cumplan los más altos requisitos de calidad y que realicen sus propósitos y objetivos.

En esta perspectiva, las universidades tendrán que construir, con la responsabilidad característica de las organizaciones del conocimiento, metodologías que permitan mostrar objetivamente, el nivel de calidad con que desarrollan sus funciones.

La elaboración de una concep- tualización, una metodología y unos procedimientosque permitan establecer cómo se desempeñan las universidades con respecto a sus funciones fundamentales de docencia, investigación y servicio a la sociedad, requiere de la participación de los académicos en la definición del marco conceptual y en la construcción del programa de trabajo de cada universidad. La misma universidad responsable de su quehacer y de la organización de sus procesos internos, es la primera interesada en establecer objetivamente la calidad con que desarrolla sus funciones $y$ en estructurar procesos de cualificación permanente a partir de esos resultados.

"La Autonomía Universitaria siempre se ha alzado como símbolo de la independencia indispensable del pensamiento en la investigación y el esclarecimiento de la verdad" (Borrero, 1994). Nadie más que la universidad es la llamada a establecer, a partir de la autoridad de la que está investida, la pertinencia, coherencia y calidad de los procesos con que asume su compromiso. Sin embargo, esa autoridad, esa responsabilidad de ser autónomo no se otorga a través de un acto formal.

Las universidades no han tenido que pedir el reconocimiento de su autonomía. Las que la han ejercido, la han me- 


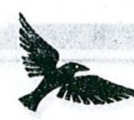

recido por el "pulcro hacer cotidiano de sus ejercicios autónomos, libres y a la vez responsables". Connatural a los ejercicios de la universidad, es el ejercicio de la autonomía. Sobre ello, desde una perspectiva teórica, es difícil admitir cualquier objeción. Es importante, sin embargo, analizar, cómo en casos concretos se hace inadecuado uso de la misma. Probablemente, en esos casos, no es la autoridad del saber académico, propia de las universidades, la que orienta su quehacer, ni la que sustenta su autonomía. El poder del saber, en el contexto deuna institución de educación superior comprometida con el conocimiento, con el hombre y con la sociedad, sí está en capacidad de señalar caminos y de orientar decisiones sobre las actividades propias de la institución, sin tener que entrar a disquisiciones con otros poderes.

La universidad, que procede de acuerdo con sus notas características como tal, es un espacio para la expresión de la libertad de cátedra, de enseñanza y de aprendizaje y de la libertad de la institución para organizar autónomamente su estructura, dirección y modos de operar en la búsqueda de la realización de su misión. Esto debe hacerlo como institución de educación superior, con todo el compromiso que conlleva la comprensión de lo que esto significa.
Reflexión pertinente en este sentido, es expresada por el Padre A. Borrero (1994). "Porque la corporación se respetaba, era respetada. Pensamos de paso, qué queda de la nota esencial autónoma si la universidad de hoy, irrespetuosa de su ser, resulta incapaz de gobernarse. Se invalida para ser autónoma. Digamos con crudeza que no merece existir tal institución porque dejó de ser lo que estaba llamada a ser: juiciosamenteautónoma. Por ello, es triste pensar que la universidad deba apelar a poderes externos para poder ordenar su casa".

$Y$ desde su exterior, se está pidiendo a la Universidad colombiana que muestre si es una casa ordenada. Undifícil punto es menester alcanzar, un punto en el que, sin menoscabar la autonomía universitaria, sin alterar el orden interno institucional, el Estado pueda, con el concurso de la comunidad académica universitaria, concebir y diseñar el Sistema Nacional de Acreditación, de modo que su razón de ser sea la de constituirse en el soporte fundamental de un sistema para el mejoramiento de la calidad. Un sistema que deje bajo la responsabilidad de las universidades la tarea de convertirse en instituciones autónomamente autorreguladas; un sistema que vaya fortaleciendo esta alternativa frente a la de adecuarse a una idea de Estado controlador y evaluador, y por lo tanto, regulador desde el exterior del quehacer de las universidades.

Este enfoque, en principio, ubica la responsabilidad de la calidad de la educación en quien realmente puede asumirla: en la institución educativa. Lo importante para el desarrollo institucional es que el mejoramiento de la calidad se viabiliza una vez la universidad asimile los resultados de un proceso de autoevaluación y reoriente su quehacer sobre la base de comprometerse con la autoevaluación como estrategia institucional, en cuanto ésta posibilita la autorregulación y, por lo tanto, su cualificación permanente.

En Colombia, la acreditación, desde la perspectiva jurídica, se plantea como un nuevo

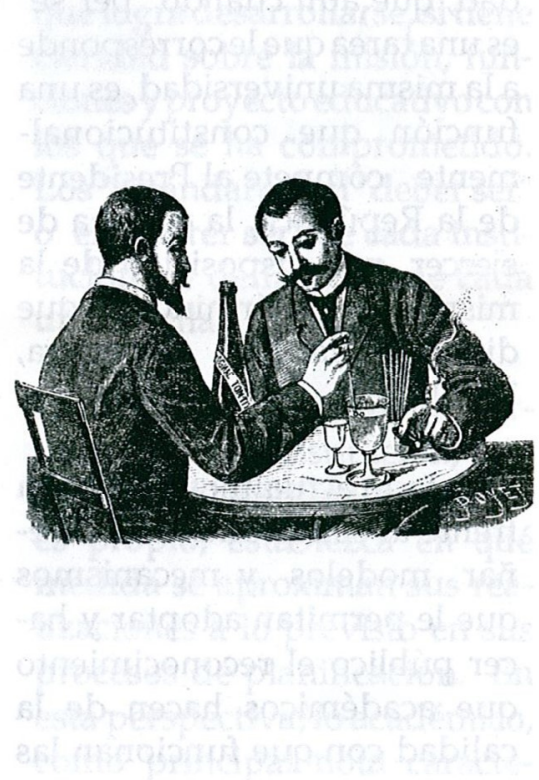




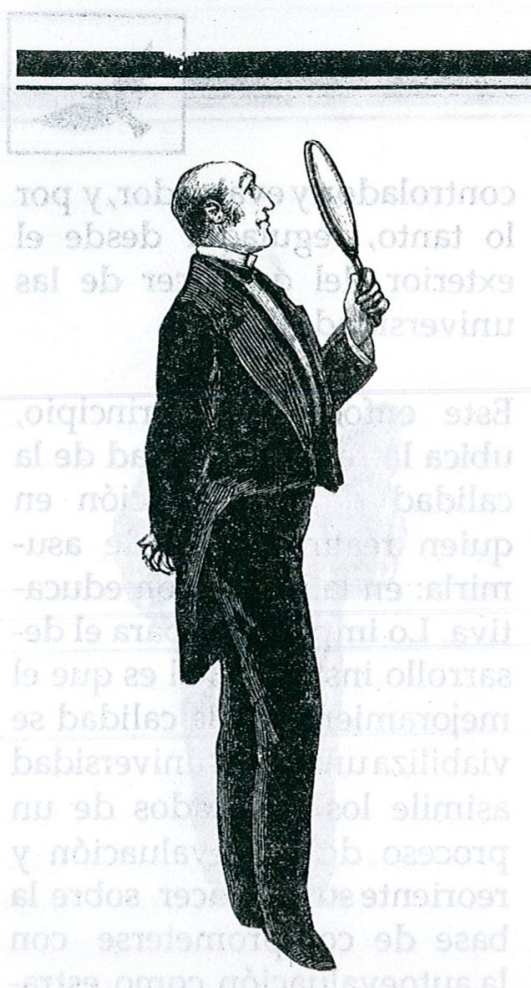

mecanismo para garantizar la calidad con que las instituciones de educación superior realizan sus propósitos y objetivos; de alguna manera "se hace referencia a una forma de inspeccionar y vigilar, a un sistema de evaluación de la calidad, que aún cuando "per se" es una tarea que le corresponde a la misma universidad, es una función que constitucionalmente compete al Presidente de la República, la cual ha de ejercer, por disposición de la misma, en los términos en que disponga la ley". (Noguera, 1996).

El Estado actualmente se halla frente al compromiso de diseñar modelos y mecanismos que le permitan adoptar y hacer público el reconocimiento que académicos hacen de la calidad con que funcionan las instituciones de educación superior; es una tarea que permite avanzar en el compromiso de garantizar a la sociedad, a través del SNA, calidad en el desarrollo de los procesos educativos. Sin embargo, en ese propósito bien se puede llegar a diseñar procedimientos que generen información abundante sobre el estado de una institución, que la describan en un momento dado en el. tiempo y que suministren bastante información cuantitativa pero relativamente insustancial frente al propósito de analizar y comprender las formas en que una institución desarrolla su misión y cumple sus funciones. En el propósito de mejoramiento de la calidad de la educación, estos procedimientos pueden llegar a ser irrelevantes si la misma institución en su autoevaluación, no ha señalado alternativas de acción que, fundadas en la comprensión de su propia dinámica, permitan avanzar en su cualificación y desarrollo permanentes.

Es decir, el conjunto de acciones de evaluación realizadas con la mira inmediata de la acreditación, sin un compromiso de la comunidad de la institución, bien puede reportar información que permita integrar a la institución al SNA o también excluírlo del mismo, sin que esto vaya acompañado deuna comprensión de la situación y prospectiva de la insti- tución. La concepción del SNA debe partir del reconocimiento de la autonomía de las universidades y de su responsabilidad social en el ejercicio de su quehacer; de esta manera, la acreditación, en instituciones conscientes de la responsabilidad desu autonomía, se genera como consecuencia de las estrategias institucionales de autoevaluación y autorregulación, cuyo fundamento $y$ finalidad es el mejoramiento de la calidad; así no se reduce solamente al acto de hacer públicas las condiciones en 'que desarrollan su misión y objetivos.

Es indudable que en la base de la creación del Sistema Nacional de Acreditación se encuentra una forma de concebir la relación Estado-Universidad, diferente a la del Estado fiscalizador y regulador que primó desde el Decreto 80 de 1980 en el país. El sistema es concebido desde la perspectiva de un Estado evaluador, que no es quien ejerce directamente la función de evaluador. El Estado, a través del SNA, debe adoptar una serie de mecanismos que permitan acudir y tener en cuenta fuentes externas de información y apreciación para validar los resultados de los procesos de autoevaluación. El Consejo Nacional de Acreditación podría concebirse como una instancia académica cuya competencia abarque la estructuración de 
un modelo que incluya estrategias técnicamente fundamentadasque permitan conceptuar sobre la validez de la autoevaluación, entre otras cosas. La estructura, organización y composición de este Consejo debe garantizar que las "instituciones, el gobierno y la opinión pública reconozcan plena legitimidad a sus actuaciones". (Brunner, 1994).

Para Brunner (Brunner, 1993), la mayoría de los "problemas que causan el malestar con que actualmente convive la educación superior, tienen su origen en la estructura de relaciones que se ha establecido entre los sistemas, la sociedad y los gobiernos durante las últimas décadas". Y entre otras, en esa estructura de relaciones es notoria la renuncia del Estado a generar mecanismos para que las mismas insti- tuciones de educación superior se autoevaluaran y se sujetaran a evaluaciones externas; lo anterior contribuyó a estimular crecimientos vegetativosde lasinstituciones, sin que se tuviese información sobre su solvencia académica. El Estado desplegó innumerables controles administralivos detallados y normas que interferían con la orientación autónoma desde dentro de las universidades; probablemente, lo anterior condujo en parte a que algunas instituciones de educación superior se liberaran de la responsabilidad con su quehacer y sus resultados; en manos del Estado estaba la tarea de garantizar que éstas cumplieran con las normas que él expedía para dirigir, regular y controlar la educación.

El nuevo tipo de relaciones entre Estado y Universidad de-

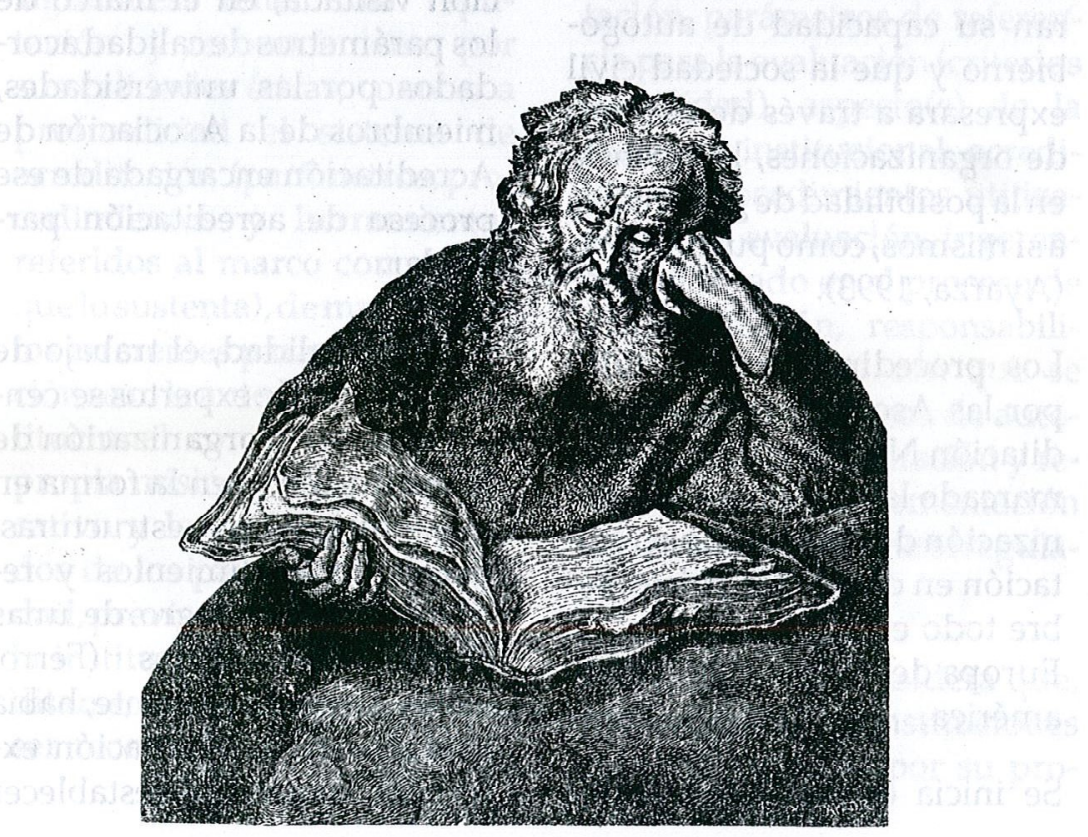

be buscar fortalecer la autonomía de las instituciones y sus capacidades de autogobierno. Y continúa Brunner: "Se supone que el Estado retira los controles administrativos minuciosos y generalmente inútiles que en la actualidad ejerce sobre las universidades y que éstas dejan de ser consideradas como parte de la administración pública... A su vez, éstas necesitarían modificar sus propias formas internas de gobierno, reforzándose en general sus estructuras de autoridad y volviéndose más ágiles de modo de generar procesos expeditos de toma de decisiones".

Las instituciones de educación superior son entonces las llamadas a organizar sus sistemas de autoevaluación. Toda institución podrá llegar a establecer el nivel de calidad con que logra desarrollarse, si tiene claridad sobre la misión, funciones y proyecto educativo con los que se ha comprometido. Los "estándares", el "deber ser" o el "querer ser" de cada institución, se definen desde cada una de ellas.

La autoevaluación debe permitir que cada Institución, desde el perfil institucional que le es propio, establezca en qué medida se aproximan sus realizaciones a lo previsto en sus procesos de planificación. En esta perspectiva, lo académico, como principal nota característica de la universidad, debe 


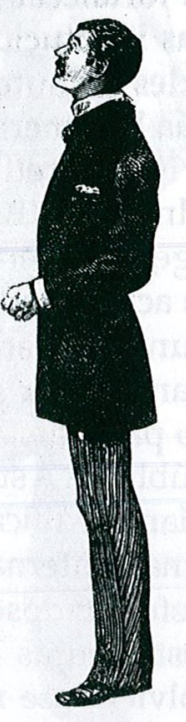

constituírse en el eje de la evaluación y en consecuencia, el trabajo de construcción de un modelo de acreditación, debe articularse sobre las funciones académicas propias de estas organizaciones.

\section{LA ACREDITACION, ANTECEDENTES $Y$ FORMAS DE ORGANIZACION}

En Norteamérica, a comienzos de siglo, varias universidades acordaron realizar una serie de actividades conjuntamente; el propósito general era buscar la calidad y desarrollar una serie de estrategias que les permitiera lograr el reconocimiento externo de la comunidad académica y de la sociedad, de que sus actividades se desarrollaban sobre la base de una búsqueda permanente de la exce- lencia, si ese era el caso. Para ello, utilizaron procesos de autoevaluación y de evaluación interinstitucional. La evaluación bien podría centrarse en los programas de la institución o en ésta como una totalidad. Se construyó y desarrolló un sistema de acreditación compartido entre ellas, a través del cual se daba crédito o constancia pública de la calidad con que las universidades asociadas funcionaban. Se llegó a organizar seis (6) asociaciones en el país, localizadas en diferentes regiones. Estas asociaciones surgieron como entes independientes del Gobierno Federal y aún hoy conservan este carácter.

La creación de estas asociaciones privadas, enraizada en la cultura y filosofía del pueblo norteamericano, permitía que las universidades demostraran su capacidad de autogobierno y que la sociedad civil expresara a través de ese tipo de organizaciones, la creencia en la posibilidad de gobernarse a sí mismos, como pueblo libre. (Ayarza, 1993).

Los procedimientos seguidos por las Asociaciones de Acreditación Norteamericanas han marcado la pauta para la organización de sistemas de acreditación en diferentes países, sobre todo en algunos países de Europa del Norte, y en Latinoamérica.

Se inicia el proceso con una primera evaluación realizada por las mismas instituciones: evalúan su organización, funcionamiento, recursos y el cumplimiento de metas, generalmente con respecto a parámetros de calidad, establecidos por las mismas universidades miembros de la Asociación que acredita.

Una segunda fase, se desarrolla a través de una visita de expertos; su tarea es la de establecer, en primera instancia, la correspondencia entre lo consignado en la autoevaluación y la realidad institucional que ellos abordan.

La visita de los expertos concluye con la presentación de un informe; para su elaboración se toman como criterios de referencia, los definidos en primera instancia por la misión y las metas de la institución visitada, en el marco de los parámetros de calidad acordados por las universidades, miembros de la Asociación de Acreditación encargada de ese proceso de acreditación particular.

En la actualidad, el trabajo de la comisión de expertos se centra más en la organización de la institución, y en la forma en que "dirige sus estructuras, planes, procedimientos y recursos hacia el logro de unas determinadas metas. (Ferro, 1994). Tradicionalmente, había primado en la evaluación externa el propósito de establecer 
la existencia y calidad de los recursos de la institución y de los programas y las fortalezas y debilidades en las diferentes áreas de desempeño. Esta aproximación podía agotarse en un estudio meramente descriptivo con bastante detalle sobre el estado de la institución en un corte transversal, sin abordar lo relacionado con la dinámica de la organización de la institución. Es la comprensión de esta dinámica la que permite allegar elementos para estimular y orientar a la institución hacia la cultura de la autorregulación.

El carácter de los expertos define en parte la posibilidad de orientar el proceso de evaluación externa, desde un punto de vista comprensivo y constructivo. Por ello, entre los criterios para la selección de los expertos se han afirmado los siguientes: tener una reputación y reconocimiento por sus calidades éticas; conocer a profundidad el sistema de acreditación (parámetros, procedimientos y herramientas referidos al marco conceptual quelosustenta), de manera que los referentes para esta evaluaciónsean la autoevaluación institucional con referencia a la propia misión y proyecto educativo y los referentes extraidos de los parámetros de calidad, previamente acogidos por la institución que quiere acreditarse; y en tercera instancia, ser reconocido como académi- co con autoridad en el campo de su formación.

El informe elaborado por la comisión de expertos se remite a la institución visitada para que emita por escrito su concepto sobre el mismo. Los dos informes se envían a la agencia acreditadora, la cual, a partir de un análisis cuidadoso, coteja las dos aproximaciones evaluativas, emite un concepto sobre la posibilidad de acreditación de la institución evaluada y entrega un informe con recomendaciones a las mismas, desde la perspectiva del interés por su mejoramiento institucional.

A partir de este esquema básico de acreditación han surgido en el contexto universitario mundial diversas alternativas que varían principalmente en lo relativo a los siguientes aspectos: objetivos de la acreditación, parámetros de referencia para la evaluación (criterios de calidad), aspecto(s) de la realidad institucional acreditados, procedimientos utilizados en la evaluación, ingerencia del Estado en el proceso de autoevaluación, responsabilidad institucional con que se asumen los procesos de autoevaluación y acreditación y relación con la implementación de procesos de autorregulación.

H. Kells (1994) considera que, por lo general, las instituciones más preocupadas por su pro- pia cualificación ponen más énfasis en la autoevaluación, en la actividad autorreguladora y en la infraestructura institucional requerida para el funcionamiento permanente de este proceso; además, afirma, dependen menos de influencias gubernamentales, tienen una visión comprehensiva e integral de las actividades de la institución y desarrollan, por lo general, estrategias que les permiten obtener retroalimentación conducente a mejorar el desempeño institucional en el ejercicio de sus funciones.

Si se da confianza a las universidades, concluye, si se les da un tratamiento de "adultos" y no de "niños", las universidades actúan con mayor madurez; entienden el significado y las implicaciones de montar un sistema de autorregulación y proceden en la búsqueda per-

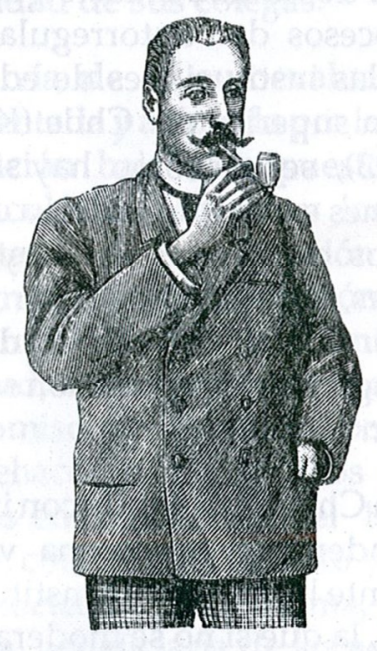




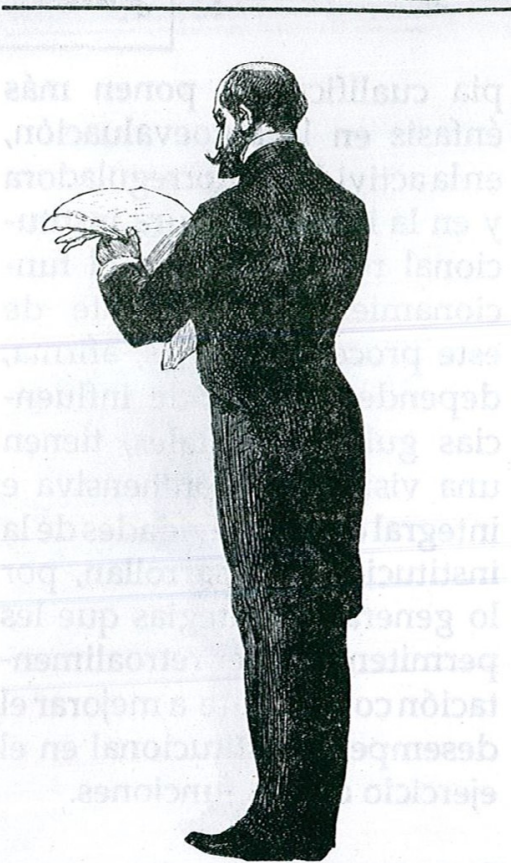

manente de patrones de excelencia con bastante independencia de sistemas de control, inspección y vigilancia externos.

Sin embargo, no desconoce la otra cara de la moneda. En el documento que elabora para desarrollar un marco conceptual de fundamentación de los procesos de autorregulación de las instituciones de educación superior de Chile (Kells, 1993), señala cómo hay situaciones en las que se deja a otros tipos de circunstancias y valores, la función de control de la proliferación y calidad de los programas de educación superior.

"En Chile se defiende con independencia y en forma vehemente la autonomía institucional, la que si no se modera por medio de un proceso de auto- rregulación responsable, puede traducirse en instituciones y programas ineficaces, un uso ineficiente de recursos escasos y una deficiente capacidad de reacción ante las necesidades globales del país y de su gente. Complican esta situación, en algunas partes del sistema, los que arguyen en favor de la aplicación descontrolada de un método de "libre mercado" al desarrollo y funcionamiento institucional, que excluya la regulación profesional sistemática y basada en la colaboración en favor de las decisiones del mercado de los clientes. En una sociedad con recursos relativamente limitados, pocos clientes (los estudiantes) tienen el tiempo y el dinero para ir de una a otra universidad en busca de la excelencia, aún si tiene la experiencia y los conocimientos (y la mayoría no los tiene) para conocer las buenas prácticas y reconocer el fraude. Sin embargo, persisten los argumentos en favor de las decisiones de mercado.

Finalmente, los chilenos no cuentan con los beneficios de un sistema eficaz de planificación del Estado para la educación superior, que en nombre de la gente atribuyan funciones (propósitos) y alcance (extensión de los programas, tipo de programas) a las instituciones. Al no existir ese sistema, proliferan los subprogramas mucho más allá de las necesidades del mercado, gas- tando recursos valiosos, y persiste una confusión inofensiva, sólo en apariencia, respecto del término "universidad". En muchos países hay universidades en las que se realiza investigación y otras que pueden o no llamarse universidades pero que existen para ofrecer una enseñanza excelente a un gran número de estudiantes. La mayoría de los países tienen además instituciones técnicas universitarias o no universitarias para preparar a técnicos y ofrecer ciertos tipos de carreras profesionales. Un número cada vez mayor de países tienen también instituciones locales o "comunitarias" organizadas para satisfacer las necesidades de zonas o comunidades específicas, para proporcionar a los estudiantes un "trampolín" flexible que les permita a la larga avanzar hacia estudios universitarios, y para abordar un nivel más local de formación técnica o profesional. Puesto que no hay un sistema diferenciado dentro de cuyos sectores las instituciones puedan alcanzar la excelencia y se las tenga en alta consideración por lograr fines y metas diferentes, todas las instituciones se esfuerzan por ser una "universidad" y muchas no tienen absolutamente ninguna posibilidad de serlo más allá que en el nombre. Ante la carencia de una política que sea aplicable $y$ que tenga sus funciones $y$ alcance bien planificados, sub- 
sistirán la confusión y la hipocresía y habrá un ámbito muy difícil para el desarrollo de una cultura de la regulación que sea eficaz y útil".

\section{LA AUTOEVALUA- CION EN LA PERS- PECTIVA DE LA AUTORREGULA- CION}

Las políticas para la orientación y el control de la Educación Superior en los últimos años en América Latina (Brunner, 1994) dejaron, como una de las consecuencias más funestas para el desarrollo de las Instituciones de Educación Superior en estos países, una cultura institucional fincada en una concepción según la cual, las aprobaciones y sanciones formales del Estado acerca del cumplimiento de requisitos para desarrollar proyectos académicos, y el seguir cuidadosamente los preceptos legales, que pretendían organizar las actividades de varios ámbitos de la vida universitaria, son indicadores objetivos y respetables de actividades propias de las universidades de calidad. Coherente con esa cultura, es el razonamiento que expresa quela existencia de instituciones y programas de deficiente calidad, es responsabilidad del Estado y aún más, la cadena continúa su desarrollo armónico, si se concibe que la acreditación es un mecanismo estatal que permite controlar la calidad de las Instituciones de Educación Superior desde el exterior.

Esta perspectiva corresponde a uno de los modelos extremos de control de la calidad. Según

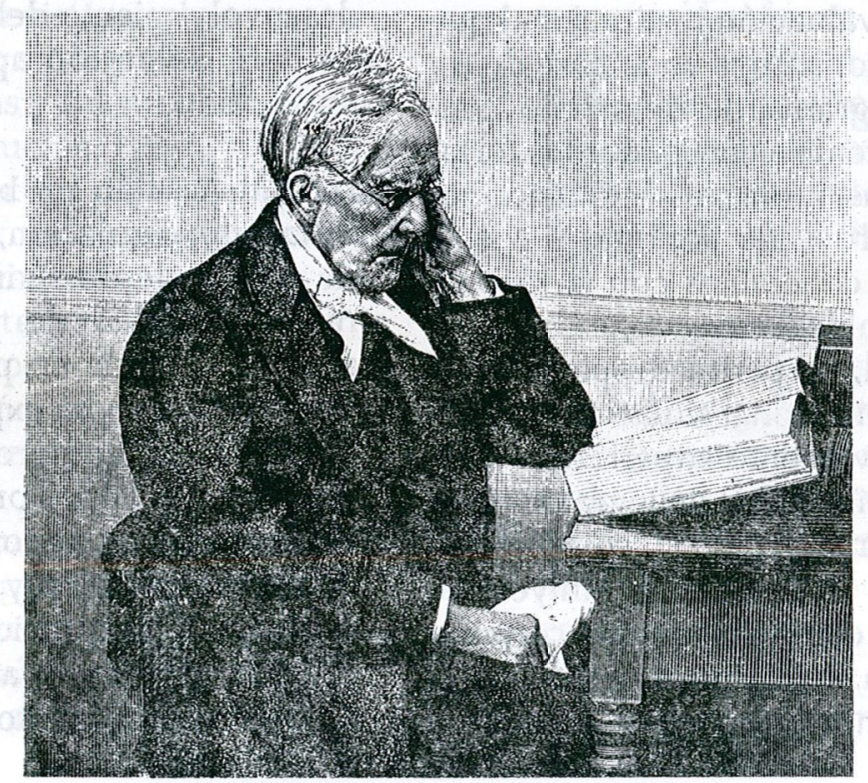

Cobban (1975) tiene su origen histórico en "la práctica francesa de conferir el control de una autoridad externa". Van Vught (1993) ilustra este enfoque con la lucha de la Universidad de París para lograr su autonomía, a comienzos del siglo XVIII, frente a la autoridad del Canciller de la Iglesia de Notre Dame, comodelegado del Obispo de París, quien autorizaba o negaba licencias para enseñar y además tenía la potestad de definir los contenidos de la enseñanza.

Otro modelo, acorde con la Universidad concebida como una corporación autónoma de socios, tiene su origen en las universidades de Oxford y Cambridge. De acuerdo con el, las Universidades debían ser instituciones completamente independientes de la jurisdicción externa. Sus socios tenían el derecho de elegir a directivos y profesores y de juzgar la calidad de sus colegas.

La vía de la autoevaluación orientada y asumida por la institución bajo el supuesto de articular el proceso y los resultados de la evaluación a la planeación y redefinición de acciones futuras de la universidad, atribuye a éstas el compromiso de responder por su quehacer en los ámbitos propios en relación con el hombre, con el conocimiento y con la sociedad. Esta última, gestora, beneficiaria de las actividades de las universidades en 


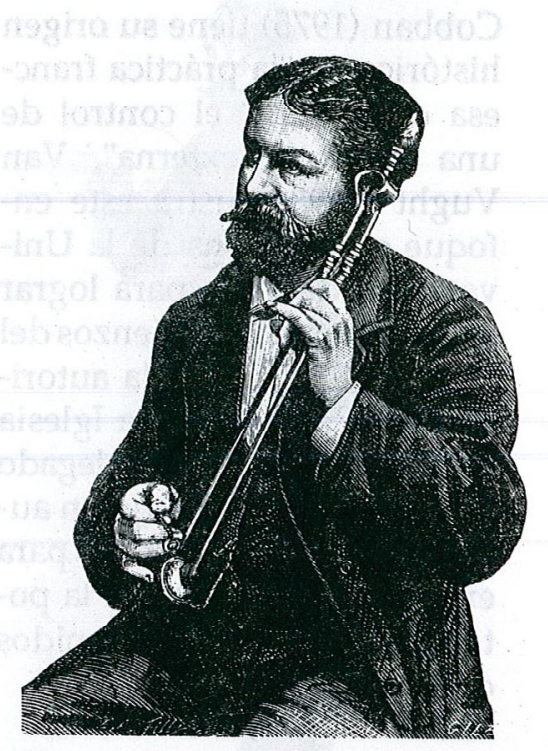

lo relacionado con la formación y la investigación, participante activa en los programas de servicio y fuente de financiación primordial para un segmento amplio de las Instituciones de Educación Superior en la actualidad, reclama un papel protagónico en la tarea de definir si estas instituciones responden a sus expectativas de calidad y eficiencia. La universidad se autoevalúa entonces, en primera instancia, en, la perspectiva de asumir la responsabilidad de cualificar sus acciones; el beneficiario de éstas es en primera instancia la sociedad.

El Estado y la sociedad, por otra parte, entrarían a asumir un rol que les permite mostrar a la sociedad en general y a los usuarios en particular, el compromiso y realizaciones de las
Instituciones de Educación Superior, en actividades pertinentes, oportunas y adecuadas a la búsqueda de la excelencia; acciones con este propósito se han concretado en lo previsto en la evaluación externa, en la cual pares académicos representan instancias externas a la institución. Proceso que puede complementar en forma significativa y constructiva el proceso de autoevaluación, asumido en forma autónoma por la institución; es el segundo paso adoptado para la acreditación con el nombre de evaluación externa.

Elementos que aporten a la definición y organización de estos dos procesos se presentan a continuación.

\section{AUTOEVALUACION:}

Solamente a partir de una autoevaluación institucional que se constituya en la base de un programa de desarrollo conducente a consolidar las fortalezas, corregir las debilidades y proyectar acciones y procesos coherentes con la realización del Proyecto de Universidad, se podrá desplazar a la institución a situaciones cualitativamente superiores. Propósito difícil de lograr si no se asume en toda su complejidad por la Institución universitaria, como una unidad corporativa. La decisión política y el compromiso para orientar $y$ apoyar el desarrollo provienen de las directivas; la construcción y consolidación de una cultura de la evaluación y de la transformación permanente involucra a todos los miembros de la comunidad universitaria; la definición de la orientación conceptual, del modelo de evaluación y de la metodología y procedimientos, también son tarea de la comunidad.

Acciones y compromisos necesarios que, sin embargo, no se dan sin un grupo que se responsabilice, lidere, organice y desarrolle el proceso.

Aún más, si en esta perspectiva autoevaluación implica transformación, surgen otros elementos que deben constituirse como inherentes y característicos de las actividades universitarias: el compromiso y la apertura frente al cambio y la innovación, siempre que el desenvolvimiento del proceso de autoevaluación apunte en ese sentido.

Una institución en búsqueda de la autorregulación, en la vía de asumir desde su interior la responsabilidad de proceder autónomamente en pro de la excelencia, deberá explorar su potencial de compromiso en relación con varios principios. De acuerdo con su concepción de autorregulación y de autoevaluación institucional, tendrá que considerar, analizar y construír un conjunto de prin- 
cipios, fundamento y orientación de la acción. "La Universidad que se autorregula (Kells, 1994) logra un nivel de autoconocimiento, que incrementa en cada ciclo de autoevaluación, y una fortaleza que acumula a través del control interno, de la regulación, del cambio y de la validación y asistencia de pares académicos y de otras instituciones, lo cual le permite afirmarse permanentemente y responder a los retos y cuestionamientos a los que se ve enfrentada".

El desarrollo de procesos de autorregulación eficaces, supone que:

- Compromete a los miembros de la comunidad universitaria y está enraizados en la institución.

- Está apoyado por sistemas de información adecuados para los procesos previstos.

- Compromete a las directivas y miembros de la institución, desde una perspectiva política, financiera y fundamentalmente académica.

- Integra necesariamente procesos de autoevaluación, de planificación y de presupuestación.

Para que la adopción de una estrategia de autorregulación culmine en programas conducentes al mejoramiento institucional, se debe garantizar que:
- Quienes tengan que poner en práctica estos programas, cuenten con posibilidades amplias de participación en las actividades de autoevaluación.

- Las opiniones de los usuarios se recojan cuidadosamente.

- La institución diseñe e incluya mecanismos para incorporar constructivamente los cambios recomendados.

- La autoevaluación se realice como un proceso de estudio cuidadoso y sistemático, con una conceptualización y procesos compartidos en su construcción y ejecución y con una perspectiva académica clara en su fundamentación y desarrollo.

- Que se cuente con los recursos financieros suficientes para poder asumir los costos de: el equipo responsable de su liderazgo y ejecución, la puesta en marcha del proyecto y el desarrollo de los programas conducentes al mejoramiento institucional.

Antes de asumir el compromiso con la autorregulación y de vincular a la comunidad con la autoevaluación, la institución debería proyectar, a partir de sus posibilidades financieras $y$ operativas, el alcance y dimensión del proceso de autoevaluación que se asume. Si no hay viabilidad de emprender las acciones del programa de mejoramiento por dificultades económicas o de otra índole, es probablemente más sano restringir el ámbito de evaluación a ciertas unidades o programas para poder desarrollar a cabalidad el proceso.

Frustración y desesperanza pueden gestarse en un proceso de autoevaluación, realizado con esfuerzo y compromiso por los participantes, si su etapa complementaria de reordenamiento no se realiza y queda en términos propositivos como sugerencias que terminan en los archivos de la institución.

Otras condiciones institucionales deben generarse como apoyo para el proceso (Kells, 1994):

- Antes de iniciar la activi-

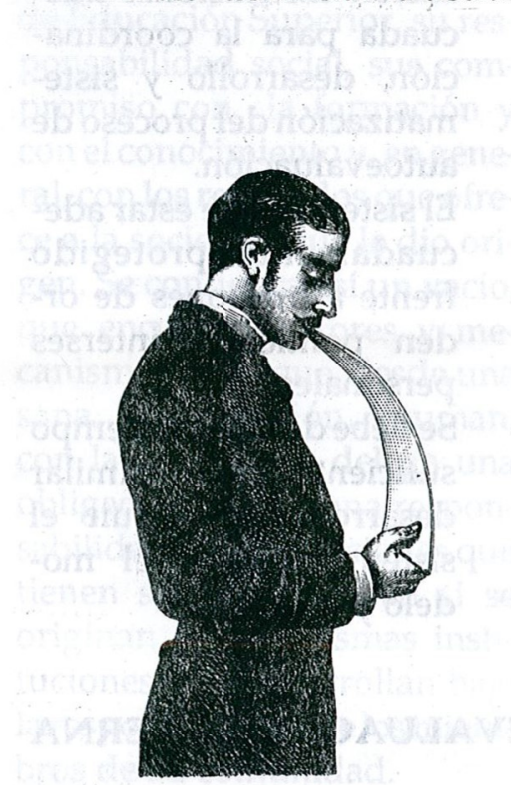




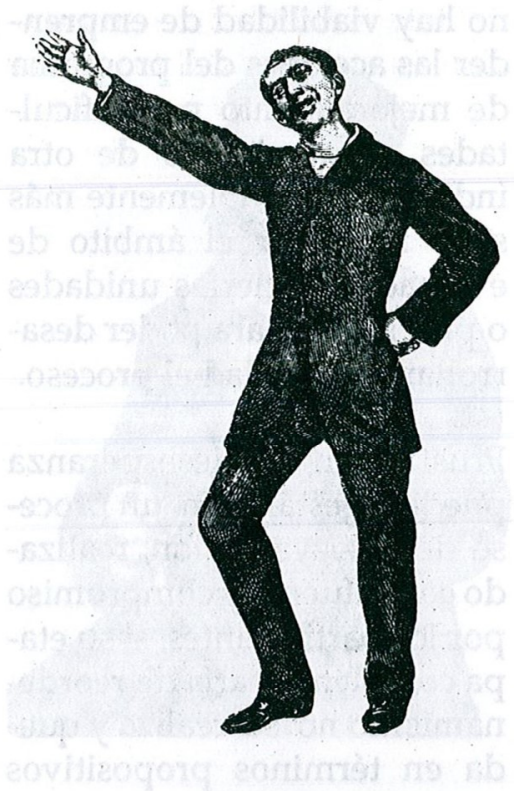

dad de autoevaluación como tal, la institución debe comprometerse con un programa para construir en forma sólida, reflexiva y participativa una cultura de la autoevaluación.

- Debe haber una organización institucional adecuada para la coordinación, desarrollo y sistematización del proceso de autoevaluación.

- El sistema debe estar adecuadamente protegido frente a presiones de orden político $o$ interses personales.

- Se debe destinar el tiempo suficiente para asimilar desarrollos, construir el sistema, adoptar el modelo y aplicarlo.

\section{EVALUACION EXTERNA}

Si bien, la evaluación externa no es estrictamente necesaria frente al propósito de mejoramiento institucional, sí hay factores que se deben analizar desde la perspectiva de su aplicación, por sus posibilidades decontribuir al fortalecimiento técnico y a la credibilidad social del proceso de autoevaluación.

Sin embargo, su implicación fundamental está en la posibilidad de dar información a la sociedad acerca de la calidad con que las universidades ofrecen el servicio de educación superior. Por otra parte, pueden constatar la medida en que las instituciones se ajustan a normas y estándares externos que buscan introducir y mantener procesos de calidad. La responsabilidad de esta evaluación debe ser también de académicos. El marco conceptual y el espíritu con los cuales se oriente el proceso no deben perder de vista el carácter y el propósito de la evaluación externa: fundamentalmente se concibe como una estrategia que permite definir si los resultados de la autoevaluación se ajustan a la realidad institucional.

La mera descripción de procesos académicos, administrativos, de los recursos e insumos de la institución y de sus resultados e impacto en la sociedad pueden quedar a nivel de información objetiva de orden cuantitativo; poco valor ten- drían resultados de este tipo para la institución y su entorno. El proyecto institucional que anima el quehacer de la universidad y la estructuración y organización de la institución y de los medios adecuados para su logro, constituyen información valiosa en el propósito de informar al público sobre la calidad de una institución y sobre su compromiso con la excelencia. Datos cuantitativos son importantes y adquieren sentido si se dan en el contexto de un marco comprensivo.

Desde el punto de vista de la credibilidad social del proceso, los resultados de la evaluación externa se constituyen en voz independiente, que permiten dar garantía a la sociedad acerca de los méritos de la institución o programa.

Desde la perspectiva de los aportes técnicos al proceso de autoevaluación en la perspectiva de la autorregulación, la evaluación externa funciona como estrategia conducente a la validación del informe de autoevaluación. Necesariamente aporta al análisis realizado por los miembros de la institución, desde su interior, y enriquece la evaluación al ampliar los marcos de referencia.

Con relación a los procesos de autorregulación, la evaluación externa, realizada desde la 
perspectiva de una comprensión institucional, permite generar programas de cualificación y fomenta e induce procesos de autorregulación, en cuanto a las mismas conclusiones o resultados que deban asimilarse, se centran en que la institución evalúe y realice sus planes, asuma las recomendaciones surgidas en el mismo proceso y readecúe condiciones institucionales en la perspectiva desus progresos.

Se pueden asimilar los dos procesos anteriores, en la perspectiva planteada por el ICFES (1994), al considerar las ventajas de cada uno e integrarlos en una visión de conjunto: "La autoevaluación posibilita la participación del personal de la institución, genera compromiso, crea una vinculación entre los evaluadores y las estructuras de decisión de la ins- titución y en principio, recibe una mayor y mejor aceptación por parte de la comunidad académica.

A su vez, la evaluación externa genera una mayor objetividad: tiene un carácter más profesional, aunque los educadores están menos comprometidos y se encuentran limitados para evaluar la cultura institucional.

De allí la utilidad de vincular los dos procesos apoyándolos en técnicas estadísticas y en metodologías no exclusivamente cuantitativas, estrategias éstas que permitirán visualizar la dinámica interna de la institución como un todo".

Del equilibrio y tipo de relaciones que se establezcan entre la evaluación desarrollada por la misma institución y los procesos de evaluación externa, se

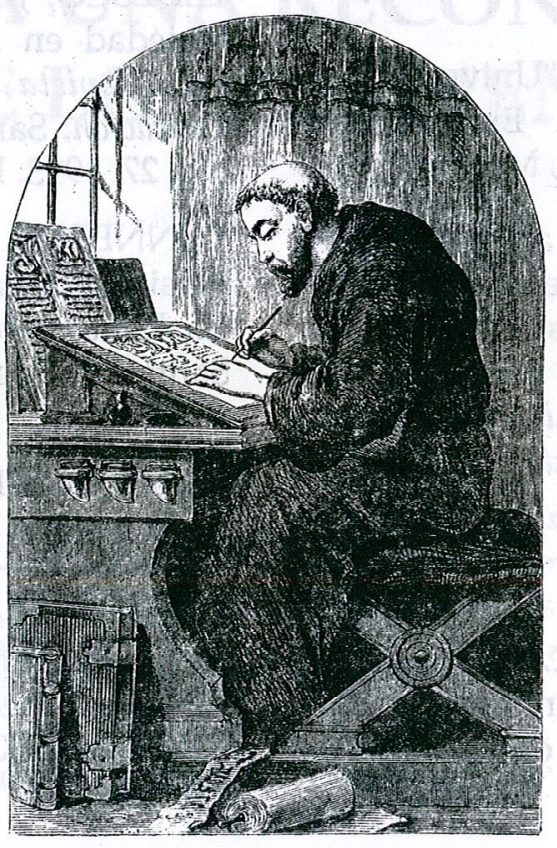

desprenderán opciones diversas para un ejercicio de la autonomía universitaria, en lo que se refiere a la capacidad de asumir el control de calidad en eldesempeño de sus funciones.

Las instituciones de educación superior, conscientes de esa responsabilidad, son las llamadas a activar mecanismos de autoevaluación y autorregulación y a desarrollarlos con objetividad, transparencia y compromiso.

Si este compromiso no surge de la misma institución, organismos ajenos a la institución, en representación del interés de la sociedad civil y ante la obligación del Estado de responder por la calidad de la Educación, encuentran un espacio propicio para que surjan inquietudes permanentes sobre el quehacer de las Instituciones de Educación Superior, su responsabilidad social, sus compromiso con la formación y con el conocimiento $y$, en general, con los resultados que ofrece a la sociedad que le dio origen. Se configura así un vacío, que encuentra actores y mecanismos para que, desde una sana preocupación, asuman, con la fuerza que delega una obligación legal y una responsabilidad social, funciones que tienen sentido tan sólo si se originan en las mismas instituciones y se desarrollan bajo la responsabilidad de los miembros de su comunidad. 
La realización de uno de los dos procesos sin el complemento del otro, no beneficia los propósitos de mejoramiento de la calidad: la realización de procesos de evaluación en el marco de parámetros internos conduce probablemente a un aislamiento institucional coherente con ausencia de preocupaciones por la pertinencia y proyección social de la universidad.

La evaluación externa, además de insuficiente, si se asume y realiza como única alternativa, también es perversa en sus implicaciones. Se podría llegar a concebir que la institución no es de calidad, porque el Estado - la sociedad civil no han puesto en práctica políticas ni programas apropiados, conducentes al control de la calidad.

La universidad misma, si no ha llegado a la mayoría de edad, si no es coherente en sus acciones con la autonomía que le es propia, se autoperpetúa en una condición de heteronomía al no asumir la responsabilidad de su proceder. La atmósfera se contamina con un aire enrarecido por la idea de que la universidad no es la responsable de la calidad de sus acciones, así ello le cueste la autonomía

\section{REFERENCIAS}

AYARZA, M. "Acreditación y calidad universitaria". VIII Congreso Bienal. Costa Rica, 1993.
BORRERO, A. La autonomía universitaria. Santafé de Bogotá, ASCUN,1994.

BRUNNER, J. J. "Evaluación y financiamiento dela educación superior en América Latina. Bases para un nuevo contrato social". En: acreditación universitaria en América Latina. Santiago de Chile, 1993.

BRUNNER, J. J. "Estado y educación superior en América Latina". En: F.A., Van Vught y otros. Prometeo encadenado. Madrid: Gedisa, 1994.

CEPAL-UNESCO. Educación y conocimiento, eje de la transformación productiva con equidad. Santiago de Chile, 1992.

COBBAN, W. The medieval universities. Their development and organization. Londres, Methuen and Co.1975. Citado por F. Van Vught.

COOMBS, P. "Universidad e investigación". En: Asterisco Cultural, No. 4, Madrid, 1991.

FERRO, J. "La acreditación en los sistemas de educación superior en los Estados Unidos y Canadá". En: Simposio sobre acreditación universitaria. Santafé de Bogotá. D.C., ICFES, 1994.

ICFES (INSTITUTO COLOMBIANO PARA EL FOMENTO DE LA EDUCACION SUPERIOR). La acreditación integral al servicio de la calidad de la educación superior. Propuesta metodológica. Santafé de Bogotá, 1994.

KELLS, H. A Guide to evaluation processes in finnish higher education. Helsinki: Ministry of education, 1994.

KELLS, H. Autorregulación en la educación superior chilena. Santiago de Chile: Consejo Superior de Educación, 1993.

NOGUERA, C. "El sistema de acreditación en Colombia. Enfoque jurídico". En Cuadernos ASCUN: Acreditación. № 2. Santaféde Bogotá, D.C., 1996.

PRESIDENCIA DE LA REPUBLICA. Elementos para la formulación de una política sobre educación superior. Santafé de Bogotá, D.C., 1995.

TOFFLER,A. El cambio de poder. Barcelona: Plaza y Janés, 1992.

TEDESCO, J. C. Educación y sociedad en América Latina. En: Revista Colombiana de Educación. Santafé de Bogotá, No. 27, 1993. Pp. 71-86.

TUNNERMANN, C. "La universidad de cara al siglo XXI". En: Reinvención de la universidad. Santafé de Bogotá, ICFES, 1994. Pp. 3-45.

VAN VUGHT. F. "Evaluación de la calidad de la educación superior: el próximo paso". En: La evaluación académica. Documentos Columbus sobre Gestión Universitaria. Vol. 2, París: CRE-UNESCO, 1993. Pp. 6589. 